\title{
Analysis of Contagion in Emerging Markets
}

\author{
Juliana de P. Filleti, Luiz K. Hotta and Mauricio Zevallos \\ University of Campinas
}

\begin{abstract}
The spread of crises from one country to another, named "contagion", has been one of the most debated issues in international finance in the last two decades. The presence of contagion can be detected by the increase in conditional correlation during the crisis period compared to the previous period. The paper presents a brief review of three of the most used techniques to estimate conditional correlation: exponential weighted moving average, multivariate GARCH models and factor analysis with stochastic volatility models. These methods are applied to analyze the contagion between the stock market of three major Latin American economies (Brazil, Mexico and Argentina) and two emerging markets (Malaysia and Russia). The data cover the period from 09/05/1995 to $12 / 30 / 2004$, which includes several crises. In general, the three methods yielded similar results, but there is no general agreement. All the methods agreed that the contagion occurred mostly during the Asian crisis.
\end{abstract}

Key words: Conditional correlation, contagion, financial crises.

\section{Introduction}

The spread of crises from one country to another, named "contagion", has been one of the most debated issues in international finance in the last two decades. Although there is a general agreement about the existence of this phenomenon and about where it occurs, there is no consensus on the definition of contagion (see Forbes and Rigobon 2001) and how to measure it or detect it. In the analyzed period, we had the Tequila effect in December 1994 in Mexico; the Asian Flu in 1997; the Russian Cold in August 1998 (including the LTCM crisis); the Brazilian Sneeze in 1999; the Nasdaq fall in April 2000 and, a little bit far from the 1990s, the Argentinian crisis at the end of 2001. The Asian crisis is not well defined because we had an initial crisis in Thailand in June, followed by a large decrease in the Indonesian market in August and finally the Hong Kong market crash on October 17, 1997. We follow Forbes and Rigobon (2002) and consider the start of the Asian crisis in October because, according to 
them, the Asian crisis started to make the headlines in the American and British newspapers only after the crash of the Hong Kong market.

There are many definitions of contagion (see, for instance Forbes and Rigobon, 2001, Dornbush et al., 2001, Corsetti et al., 2002) but no definition is universally accepted and, as a consequence, there are many ways to measure it (see, for instance, Rigobon, 2001). One of the earliest definitions of contagion, the "shiftcontagion", regards contagion as a shift or change in how shocks spread from one country to another between "normal" periods and "crisis" periods. One way to measure it is through the conditional correlation between the return of some market indices. Although there are some criticisms about this definition and about the use of this measure (Pesaran and Rick, 2005), it has been used in many works because it can at least indicate the presence of contagion and be used as an exploratory tool. This definition was used by many authors; for instance, by King and Wadhwani (1990), Baig and Goldfajn (1998), Edwards (1998), Boyer et al. (1999), Bae et al. (2000), Loretan and English (2000), Corsetti et al. (2002), Lopes and Migon (2002), Forbes and Rigobon (2002) and Marçal and Valls Pereira (2005). Some of these authors consider that there is evidence of contagion whenever there is a shift in the correlation, for instance, Lopes and Migon (2002) and Marçal and Valls Pereira (2005). We consider that there is evidence of contagion only when there is an increase in the correlation during crisis periods.

This paper has two objectives: to briefly present some estimators for the conditional correlation of the time series data and to apply them to measure the contagion between some emerging Latin American markets (Argentina, Brazil and Mexico) and two other emerging markets (Russia and Malaysia). Contrarily to Forbes and Rigobon (2002), who only test for contagion in the country from where the shock originates and spreads to other countries, we consider the possibility of contagion between countries from where the shocks did not originate. In doing so, we consider that a shock originating from one country can increase the correlation between two other countries. For instance, we will see that the Asian crisis increased the correlation between the Brazilian and Mexican stock market indices. Although this could not be strictly a contagion, we will call it a contagion between these two countries for lack of a better name for it. In Section 2, we present the exponential smoothing method, some multivariate GARCH models and the factorial model using stochastic volatility models. The data set, the exploratory analysis and filtering are presented in Section 3. The estimation of the correlation of (conditional) volatilities by different methods are presented in Section 4. Section 5 concludes. 


\section{Methods for the Estimation of Conditional Correlation}

Let $r_{t}=\left(r_{t, 1}, \ldots, r_{t, N}\right)^{\top}$ be a multivariate time series of returns, $t=1, \ldots, T$. Assume that

$$
\begin{array}{r}
r_{t}=\varepsilon_{t} H_{t} \\
\varepsilon_{t} \sim \operatorname{IID}(0, I)
\end{array}
$$

where $I$ is the identity matrix, $\varepsilon_{t}$ are independent of past values of $H_{t}$, and $H_{t}$ is a constant conditioned on $\mathcal{F}_{t-1}$, the information available up to time $(t-1)$. Then $E\left(r_{t} \mid \mathcal{F}_{t-1}\right)=0$ and $\operatorname{Var}\left(r_{t} \mid \mathcal{F}_{t-1}\right)=H_{t}$ is the conditional covariance matrix of $r_{t}$. The elements of $H_{t}$ are denoted by: $h_{i, t}$ for the conditional variances of the $i$-th return and $h_{i j, t}$ for the conditional covariance of the $i$-th and $j$-th returns. This section presents some methods used to estimate the conditional correlation, i.e. for estimating $\rho_{i j, t}=h_{i j, t} / \sqrt{\left.h_{i, t} h_{j, t}\right)}$. We always use the hat in order to denote estimator or estimate.

\subsection{Exponential smoothing method}

In this method the conditional variance of the $i$-th series is evaluated recursively through:

$$
\hat{h}_{i, t}=\lambda_{i} \hat{h}_{i, t-1}+\left(1-\lambda_{i}\right) r_{i, t}^{2}, 0<\lambda_{i}<1,
$$

where $0<\lambda_{i}<1$ is the smoothing parameter for the $i$-th series. Denoting the estimate for the initial variance by $\hat{h}_{i, 0}$, the estimates can be rewritten as:

$$
\hat{h}_{i, t}=\lambda_{i}^{t} \hat{h}_{i, 0}+\left(1-\lambda_{i}\right) \sum_{j=0}^{t-1} \lambda^{j} r_{i, t-j}^{2}
$$

showing that $\hat{h}_{i, t}$ is the weighted mean of present and past squared returns with weights decaying exponentially to zero on observations further back into the past history. A way to estimate the initial variance is to start the estimation at time $t_{1}$ and consider $\hat{h}_{t_{1}, 0}$ as the sample variance using the first $t_{1}$ observations or to use the sample variance of the whole series.

An alternative way is to estimate $h_{i t}$ as:

$$
\hat{h}_{i, t}=\left(1-\lambda_{i}\right) \sum_{j=0}^{\infty} \lambda^{j} r_{i, t-j}^{2},
$$


where the square of the returns $r_{i, t-j}^{2}$ must be estimated for $j \geq t$.

The estimate of the covariance between two time series at time $t, h_{i j, t}$, is evaluated similarly to (2.2) as:

$$
\hat{h}_{i j, t}=\lambda_{i j}^{t} \hat{h}_{i j, 0}+\left(1-\lambda_{i j}\right) \sum_{k=0}^{t-1} \lambda^{k} r_{i, t-k} r_{j, t-k} .
$$

The initial covariance can also be estimated similarly by the sample covariance of all data or of the initial observations. We also have similar formulae to (2.1) and (2.3).

The constants $\lambda_{i}$ and $\lambda_{i, j}$ can be chosen in an ad hoc way using the knowledge of the market and of the method; for instance, a value in the interval $(0.94 ; 0.97)$ is generally recommended in the literature. Another approach is to select the parameters based on some criteria. In this case, it is common to use a criterion based on the one-step ahead prediction error. The basic idea of the exponential smoothing method is that the variable of interest changes randomly. Thus, we could use the present estimate of the volatility, i.e. $\hat{h}_{i, t}$, as the one step-ahead prediction of the volatility. The conditional volatility $h_{t+1}$ is unobservable and a proxy for the one step-ahead prediction error is given by $r_{i, t+1}^{2}-\hat{h}_{i, t}$. Thus, for the $i$-th series we select $\lambda_{i}$, which minimizes the weighted squared prediction error given by

$$
W M S E_{i}=\sum_{t=t_{0}}^{T} w(t)\left(r_{i, t}^{2}-\hat{h}_{i, t-1}\right)^{2},
$$

where $w(t)$ can be used in order to give more importance to most recent observations.

Engle (2002) suggested the use of the same value of $\lambda$ for all series, using a weighted value, but here we will select different values for each variance and covariance.

The correlation between the $i$-th and $j$-th series at time $t$ is estimated by:

$$
\hat{\rho}_{i j, t}=\frac{\hat{h}_{i j, t}}{\sqrt{\hat{h}_{i, t} \hat{h}_{j, t}}} .
$$

The S+FinMetrics function presents an alternative way to choose the smoothing parameter (see Zivot and Wang 2002, Section 13.2). Denote the return vector by $r_{t}$ and its conditional (to the past information) covariance matrix by $\Sigma_{t}$. Then, 
the quasi-log likelihood, considering the multivariate normal distribution and the mean of the returns equal to zero, can be written as:

$$
\log L=-\frac{N T}{2} \log (2 \pi)-\frac{1}{2} \sum_{i=1}^{T}\left|\Sigma_{t}\right|-\frac{1}{2} \sum_{i=1}^{T} r_{t}^{\prime} \Sigma_{t}^{-1} r_{t} .
$$

The $\mathrm{S}+$ FinMetrics uses the same smoothing parameter to estimate the variances and covariances, denoting it by $\lambda$. The coefficient $\lambda$ is treated as a parameter and it is estimated as the value that maximizes the log likelihood when the covariance $\Sigma_{t}$ is recursively calculated using (2.2) and (2.4) in the ewma1 program option. In another program option, ewma2, (2.2) is substituted by the alternative formula (2.3) and the covariance is estimated by (2.4) modified in a similar way.

\subsection{Multivariate GARCH models}

We tested different versions of multivariate GARCH models, the Bollerslev (1990) Constant Conditional Correlation (CCC) Model, The Baba, Engle, Kraft and Kroner BEKK (Engle and Kroner, 1995) model and the Engle (2002) Dynamic Conditional Correlation Model (DCC). In this section, we briefly present some results for these models. Specifically, definitions, estimation, and lack of a specification test.

\section{Constant conditional correlation (CCC) model}

In this model, proposed by Bollerslev (1990), the conditional variances are assumed to follow univariate $\operatorname{GARCH}(p, q)$ models,

$$
h_{i t}=\omega_{i}+\sum_{l=1}^{p} \alpha_{i l} r_{i, t-l}^{2}+\sum_{s=1}^{q} \beta_{i s} h_{i, t-s}, \quad i=1, \ldots, N
$$

but the conditional correlation between returns $i$ and $j, \rho_{i j}$, are considered time invariant. Then, the conditional covariances are defined as

$$
h_{i j, t}=\rho_{i j} h_{i t}^{1 / 2} h_{j t}^{1 / 2} \quad i, j=1, \ldots, N,
$$

with a total number of parameters in the model equal to $N(1+p+q)+(N-$ 1) $N / 2$. The constraints $\sum_{l=1}^{p} \alpha_{i l}+\sum_{s=1}^{q} \beta_{i s}<1$ for $i=1, \ldots, N$ are sufficient to guarantee second-order stationarity.

\section{Baba, Engle, Kraft and Kroner (BEKK) model}

This model was initially proposed by Yoshi Baba, Robert Engle, Dennis Kraft and Ken Kroner, hence the acronym BEKK, but the published updated version 
of this article includes only two of the original authors (Engle and Kroner, 1995). The objective was to define a model capable of reproducing the time-varying correlations observed in empirical studies.

In the Full BEKK $(p, q)$ model the conditional variance matrix is defined as:

$$
H_{t}=\Omega+\sum_{l=1}^{p} A_{l} r_{t-l} r_{t-l}^{\top} A_{l}^{\top}+\sum_{s=1}^{q} B_{s} H_{t-1} B_{s}^{\top}
$$

where $\Omega, A_{l}, B_{s}$ are $N \times N$ matrices of parameters with $\Omega$ symmetric and positive definite. Since the terms involving matrices $A^{\prime}$ s and $B^{\prime}$ s on the right hand side of (2.6) are quadratic forms, $H_{t}$ is guaranteed to be positively defined. Therefore, we do not need additional conditions on the parameters. As usual, conditional correlations are defined as $\rho_{i j, t}=h_{i j, t} / \sqrt{h_{i, t} h_{j, t}}$ for $i, j=1, \ldots, N$.

For Full BEKK models the number of parameters is $(p+q) N^{2}+N(N+1) / 2$. It is possible to obtain simpler versions by considering $A^{\prime} \mathrm{s}$ and $B^{\prime} \mathrm{s}$ as diagonal matrices, the Diagonal BEKK models, or scalars, the Scalar BEKK models. The number of parameters is reduced to $(p+q) N+N(N+1) / 2$ and $(p+q)+N(N+1) / 2$ for diagonal and scalar cases, respectively.

\section{Dynamic conditional correlation (DCC) model}

This model was proposed by Engle (2002) and preserves the parsimony of individual univariate GARCH models volatility for each time series with a simple time-varying correlation.

The model presents an improvement compared with BEKK models because the order of the number of parameters decreases to $k$ and the order of the number of parameters that needs to be estimated simultaneously decreases to one (Engle and Sheppard, 2001).

In DCC models, the conditional covariance matrix is defined as

$$
H_{t} \equiv D_{t} R_{t} D_{t},
$$

where $D_{t}$ and $R_{t}$ are $N \times N$ matrices. $R_{t}$ is the time-varying correlation matrix with elements $\rho_{i j, t}$, and $D_{t}$ is a diagonal matrix with elements given by the conditional standard deviations $h_{i, t}^{1 / 2}$.

Conditional variances, $h_{i t}$, are modelled as (2.5) and conditional correlations are calculated by

$$
\rho_{i j, t}=\frac{\delta_{i j, t}}{\sqrt{\delta_{i i, t} \delta_{j j, t}}} \quad i, j=1, \ldots, N
$$

where $\delta_{i j, t}$ are elements of matrix $\Delta_{t}$. This evolves by following

$$
\Delta_{t}=S(1-\alpha-\beta)+\alpha D_{t}^{-1} r_{t} r_{t}^{\top} D_{t}^{-1}+\beta \Delta_{t-1},
$$


where $S$ is the unconditional correlation matrix of $\varepsilon_{t}=D_{t}^{-1} r_{t}$.

Once the conditional variances have been determined, note that we just need two parameters, $\alpha$ and $\beta$, in order to calculate the conditional correlations. Thus, the total number of parameters is equal to $N(1+p+q)+2$, i.e, of order $N$. In addition, as shown by Engle and Sheppard (2001), the conditional variance is positively defined.

The necessary and sufficient condition for second-order stationarity in (2.7) is $\alpha+\beta<1$. However, when $\alpha+\beta=1$, we obtain a strictly stationary model. This version is named Integrated Dynamic Conditional Correlation (IDCC) model.

\section{Estimation}

Assume now that $r_{t}$ has a conditional multivariate Gaussian distribution, i.e. $r_{t} \mid \mathcal{F}_{t-1} \sim N\left(0, H_{t}\right)$. Then the log likelihood can be written as:

$$
L \simeq-\frac{1}{2} \sum_{t=2}^{T}\left(N \log (2 \pi)+\log \left|H_{t}\right|+r_{t}^{\top} H_{t}^{-1} r_{t}\right)
$$

In expression (2.8), the first term in the sum is assumed to be zero. With hundreds or thousands of observations, the effect of this term is almost null.

In order to estimate the parameters, maximization of the likelihood is done by using numerical routines, for example, Davidon-Fletcher-Powell (Fletcher and Powell, 1963) or BHHH (Berndt et al., 1974) algorithms.

It is worth saying that expression (2.8) holds for all specifications of the multivariate Gaussian model above. But, for DCC models, we can obtain

$$
L=-\frac{1}{2} \sum_{t=1}^{T}\left(N \log (2 \pi)+2 \log \left|D_{t}\right|+\log \left|R_{t}\right|+r_{t}^{\top} D_{t}^{-1} R_{t}^{-1} D_{t}^{-1} r_{t}\right) .
$$

For DCC models, sufficient conditions for the consistency and asymptotic normality of this estimator are based on Newey and McFadden (1994) results. Unfortunately, the properties of the maximum likelihood estimators of BEKK models are still not completely known (Engle and Kroner, 1995).

It is possible to deal with other conditional distributions rather than the multivariate Gaussian distribution. In this case, we still maximize (2.8) and obtain quasi-maximum likelihood estimates.

\section{Specification testing}

The robust test of conditional moment test presented by Wooldridge (1990), which assesses whether the fitted model adequately explains the data dynamics, is briefly described in this subsection. The test is useful to detect whether there 
are other variables which are relevant in explaining the residuals (Cappielo et al., 2003, Mar 蓷 1 and Valls Pereira, 2005). In order to apply the test, one should initially define a generalized residual $u_{i j, t}$, which is defined as any function of the residuals with an expectation equal to zero. We are going to use $u_{i, t}=$ $\varepsilon_{i, t}^{2} / h_{i, t}-1$ as the generalized residual. We also have to select sets of variables, also called moments, which are expected to explain the residuals. The choice of this set of variables depends on the specific lack of specification we want to test. In subsection 4.2.1 we have examples of three set of variables (equations (4.1) to (4.3)). We follow the procedure proposed by Mar 蓷 1 and Valls Pereira (2005). Denote the $g$-th set of $Q$ moments by $\lambda_{g, t-1}=\left\{\lambda_{g, 1, t-1}, \cdots, \lambda_{g, Q, t-1}\right\}$. The specification test for an $i$-th series is given by the following steps:

1. Using a consistent estimate of the model parameters, evaluate the generalized residuals, $u_{t}=\left(u_{1, t}, \cdots, u_{N, t}\right)^{\top}$; the gradient in relation to the parameter $\theta_{j}$ given by $y_{i j t}=E\left(\partial u_{i, t} / \partial \theta_{j}\right)$; and the set of moments $\lambda_{g, t-1}$.

2. Regress the conditional moments $\lambda_{g, k, t-1}, k=1, \cdots, Q$, the response variable, on the gradients $\left\{y_{i j t}, i=1, \cdots, N,: j=i_{1}, \cdots, i_{M^{\prime}}\right\}$, for $k=1, \cdots, Q$, the predictors. Calculate this regression residuals, say $\xi_{k, t-1}$. The set of values $\left\{j=i_{1}, \cdots, i_{M^{\prime}}\right\}$ selects the cases where the gradients are not zero.

3. The next step consists of a new regression, taking a vector of ones as the response variable and $\left\{u_{i t} \xi_{1, t-1}, \cdots, u_{i t} \xi_{Q, t-1}\right\}$ as regressors, product of the generalized residuals defined in step (1) with the residuals $\xi_{k, t-1}$ evaluated in the regression from the previous step. There is lack of specification when the regressors are relevant.

4. We can use $R_{u}=T-S S R$, where $T$ is the number of observations, and SSR is the sum of squared residuals of this second regression, as the test statistic. Under very mild conditions and under the null hypothesis of no lack of specification, this statistic is asymptotically distributed as a chisquare with $Q$ degrees of freedom (Cappielo et al., 2003).

The conditional moments can be any function of any variable allowing us to use this procedure to test against any form or cause of bad specification.

\subsection{Factorial analysis}

When modeling several financial time series, some of them will usually be affected by common effects. For this reason, it is reasonable to model the multivariate conditional covariance matrix of these series using factor models.

Considering that stochastic volatility models can be easily generalized to multivariate distributions (Harvey et al., 1994), it is quite natural to use them to 
model the factors. This model was initially presented by Aguilar and West (2000) and Chib et al. (2001) and applied by other authors to analyze the returns of some emerging markets (see, for instance, Lopes and Migon, 2002 and Tsai and Hotta, 2005).

\section{The factor model}

Considering the $N$-variate returns series $r_{t}$, the factor model is given by:

$$
r_{t}=\theta_{t}+X_{t} f_{t}+\varepsilon_{t}
$$

with $t=1, \ldots, T ; \theta_{t}$, an $N \times 1$ mean vector; $X_{t}$, the $N \times k, k<N$, the loading matrix; $f_{t}$, the $k \times 1$ common factors; and $\varepsilon_{t}$, an $N \times 1$ specific disturbance vector. In the literature $\theta$ and $X$ are generally taken as time invariant. We used time-varying mean and loading matrix for modeling because they produced a smoother estimate of the covariance matrix. The processes $\left\{f_{t}\right\}$ and $\left\{\varepsilon_{s}\right\}$ are independent. Conditioned on their diagonal variance matrix $H_{t}$, the factors $f_{t}$ can be considered serially independent realizations of a latent process, i.e.

$$
f_{t} \mid H_{t} \sim \mathcal{N}_{k}\left(0 ; H_{t}\right)
$$

Similarly, the specific factors, conditioned on their diagonal variance matrix $\Psi_{t}$, can also be considered serially independent realizations of a latent process, i.e.

$$
\varepsilon_{t} \mid \Psi_{t} \sim \mathcal{N}_{N}\left(0 ; \Psi_{t}\right)
$$

Thus, conditioned on $\theta_{t}, X_{t}, H_{t}$ and $\Psi_{t}$ the covariance matrix of the returns is given by:

$$
\Sigma_{t}=X_{t} H_{t} X_{t}^{\top}+\Psi_{t}
$$

and we have $r_{t} \mid\left(\theta_{t}, X_{t}, H_{t}, \Psi_{t}\right) \sim \mathcal{N}_{N}\left(\theta_{t} ; \Sigma_{t}\right)$. Therefore, by conditioning the returns series on common factors we also have a serially independent Gaussian process $r_{t} \mid\left(\theta_{t}, X_{t}, f_{t}, \Psi_{t}\right) \sim \mathcal{N}_{N}\left(\theta_{t}+X_{t} f_{t} ; \Sigma_{t}\right)$. This leads to the conclusion that common factors explain all the dependence structure among the series. The models for $f_{t}$ and $\varepsilon_{t}$ will be presented in the next subsection.

The model given by (2.9) is not identified. So, in order to have a single decomposition in (2.12), some restrictions are imposed on matrix $X_{t}$. This matrix 
must have full rank, be invariant under invertible linear transformation of the factors and must have $k$, the number of column, such that the system given by equation (2.12) is not undetermined (Aguilar and West, 2000).

We use the hierarchical form for the loading matrix $X_{t}$, as proposed by Geweke and Zhou (1996), i.e with restrictions $x_{i j, t}=0$ for $i<j$ and $x_{i i, t}=1$, in order to get the necessary restrictions of full rank and invariance. Thus:

$$
X_{t}=\left[\begin{array}{cccc}
1 & 0 & \ldots & 0 \\
x_{21, t} & 1 & \ldots & 0 \\
\vdots & \vdots & \vdots & \vdots \\
x_{k 1, t} & x_{k 2, t} & \ldots & 1 \\
x_{k+11, t} & x_{k+12, t} & \ldots & x_{k+1 k, t} \\
\vdots & \vdots & \vdots & \vdots \\
x_{N 1, t} & x_{N 2, t} & \ldots & x_{N k, t}
\end{array}\right]
$$

This means that the first return series, $r_{1}$ is modeled by the mean $\theta_{1}$ plus the factor $f_{1}$ and the specific disturbance $\varepsilon_{1}$; the second, $r_{2}$, is modeled by $\theta_{2}+$ $x_{21} f_{1}+f_{2}+\varepsilon_{2}$ and so on and so forth. Aguilar and West (2000) claim that, when using the hierarchical form for matrix $X$, the order of the series in the vector $r_{t}$ is important to the interpretation of the estimated values of $X$, to adjustment of the model, and also to the determination of the factor number $k$, but does not interfere in model forecasting because the variance and covariance are independent of the choice of the order.

Another restriction on $k$, which gives the model size, is that the number of equations $N(N+1) / 2$ must be greater than $N k+N-k(k-1) / 2$, the number of parameters in equation (2.12). We also should take into account that a smaller $k$ means a more parsimonious model.

\section{Stochastic volatility components}

The variances of common factor $f_{t}$ and the specific effects $\varepsilon_{t}$, which are the diagonal elements of matrix $H_{t}$ in (2.10) and $\Psi_{t}$ in (2.11), are respectively modeled by stationary first-order stochastic volatility models.

Denoting the $\log$ volatility of common factors $f_{t}$ as $\lambda_{i, t}^{f}=\log h_{i, t}$, for $i=$ $1, \ldots, k$, and $\lambda_{t}^{f}=\left(\lambda_{1, t}^{f}, \ldots, \lambda_{k, t}^{f}\right)^{\top}$ we have

$$
\lambda_{t}^{f}=\mu^{f}+\Phi^{f}\left(\lambda_{t-1}^{f}-\mu^{f}\right)+\eta_{t}^{f},
$$

where $\eta_{t}^{f} \sim \mathcal{N}_{k}\left(0 ; \Sigma_{\eta}^{f}\right)$ with $t=1,2, \ldots$ are independent. Denote the mean of $\lambda_{t}^{f}$ by $\mu^{f}=\left(\mu_{1}^{f}, \ldots, \mu_{k}^{f}\right)^{\top}$, the persistence by $\Phi^{f}=\operatorname{diag}\left(\phi_{1}^{f}, \ldots, \phi_{k}^{f}\right)$ and the 
innovation variance matrix by $\Sigma_{\eta}^{f}$. The matrix $\Sigma_{\eta}^{f}$ is full, i.e. it can have all the elements different from zero in order to allow for correlations among the $k$ process innovations at time $t$. Notice that $\lambda_{t}^{f} \mid\left(\lambda_{t-1}^{f}, \mu^{f}, \phi^{f}, \Sigma_{\eta}^{f}\right) \sim \mathcal{N}\left[\mu^{f}+\right.$ $\left.\Phi^{f}\left(\lambda_{t-1}^{f}-\mu^{f}\right) ; \Sigma_{\eta}^{f}\right]$.

For the specific disturbances we have $\lambda_{i, t}^{\varepsilon}=\log \psi_{i, t}$ and $\lambda_{t}^{\varepsilon}=\left(\lambda_{1, t}^{\varepsilon}, \ldots, \lambda_{N, t}^{\varepsilon}\right)^{\top}$. The log volatility process is given by:

$$
\lambda_{t}^{\varepsilon}=\mu^{\varepsilon}+\phi^{\varepsilon}\left(\lambda_{t-1}^{\varepsilon}-\mu^{\varepsilon}\right)+\eta_{t}^{\varepsilon},
$$

where $\eta_{t}^{\varepsilon} \sim \mathcal{N}_{N}\left(0 ; \Sigma_{\eta}^{\varepsilon}\right)$ are independent. Denote the mean of $\lambda_{t}^{\varepsilon}$ by $\mu^{\varepsilon}=\left(\mu_{1}^{\varepsilon}, \ldots\right.$, $\left.\mu_{N}^{\varepsilon}\right)^{\top}$, the persistence by $\Phi^{\varepsilon}=\operatorname{diag}\left(\phi_{1}^{\varepsilon}, \ldots, \phi_{N}^{\varepsilon}\right)$ and the innovation variance matrix by $\Sigma_{\eta}^{\varepsilon}$, which is taken as diagonal, i.e $\Sigma_{\eta}^{\varepsilon}=\operatorname{diag}\left(\sigma_{\eta, j}^{2}\right)$. This is the same as considering an $N$ univariate process with conditional distribution $\lambda_{t}^{\varepsilon} \mid\left(\lambda_{t-1}^{\varepsilon}, \mu^{\varepsilon}, \phi^{\varepsilon}\right.$, $\left.\Sigma_{\eta}^{\varepsilon}\right) \sim \mathcal{N}\left[\mu^{\varepsilon}+\phi^{\varepsilon}\left(\lambda_{t-1}^{\varepsilon}-\mu^{\varepsilon}\right) ; \Sigma_{\eta}^{\varepsilon}\right]$.

\section{Priors}

The prior distribution of the vector of parameter $\left\{\theta, X, \Phi^{f}, \Sigma_{\eta}^{f}, \mu^{f}, \Phi^{\varepsilon}, \Sigma_{\eta}^{\varepsilon}, \mu^{\varepsilon}\right\}$ is given by the product of independent priors:

$$
p(\theta) p(X) p\left(\Phi^{f}\right) p\left(\Sigma_{\eta}^{f}\right) p\left(\mu^{f}\right) p\left(\Phi^{\varepsilon}\right) p\left(\Sigma_{\eta}^{\varepsilon}\right) p\left(\mu^{\varepsilon}\right),
$$

where $p(\omega)$ denotes the probability density function of random variable $\Omega$ at point $\omega$. Table 1 shows the priors of $\theta, X, \mu^{f}$ and $\mu^{\varepsilon}$. They are centered on the values obtained by factorial approximation applied to part of the initial observations as proposed by Aguilar and West (2000). We use Beta distribution as priors for $\phi^{\ddagger}=2 \times \phi-1$, where $\phi$ represents each element in $\phi^{f}$ and $\phi^{\varepsilon}$. The priors of other parameters are taken from the literature (Aguilar and West, 2000, Chib et al., 2001 and Tsai and Hotta, 2005).

Table 1: Prioris for the MF-VE model, $\hat{z}$ denotes the estimate of factorial approximation to $z$.

\begin{tabular}{llcc}
\hline Parameter & Priori & Mean & Standard Deviation \\
\hline$\theta_{i}$ & $\mathcal{N}\left(\hat{\theta}_{i} ; 25\right)$ & $\hat{\theta}_{i}$ & 5 \\
$x_{i j}$ & $\mathcal{N}\left(\hat{x}_{i, j} ; 25\right)$ & $\hat{x}_{i, j}$ & 5 \\
$\mu_{j}^{f}$ e $\mu_{i}^{\varepsilon}$ & $\mathcal{N}(\hat{\mu} ; 25)$ & $\hat{\mu}$ & 5 \\
$\phi^{\ddagger}$ & Beta $(21 ; 1.5)$ & 0.86 & 0.11 \\
$\sigma_{\eta, i}^{\varepsilon}$ & IGamma $(2.4 ; 0.35)$ & 0.25 & 0.40 \\
$\Sigma_{\eta(2 \times 2)}^{f}$ & IWishart $(6 ; 0.10 \times I)$ & $0.20^{*}$ & $0.28^{*}$ \\
\hline \multicolumn{4}{c}{$*$ diagonal values of $\Sigma_{\eta}^{f}$} \\
\end{tabular}


We did not find contagion between Latin American countries during the Mexican crisis. Calvo and Reinhart (1996) argued in favor of contagion among the large Latin American countries because they detected an increase of comovements across weekly equity and Brady bond returns for emerging markets in Latin American after the Mexican crisis. However, Forbes and Rigobon (2002) found that the evidence of contagion from Mexico to the Latin America countries measured by the increase of the correlation disappeared when corrected for bias.

More details about the application of MCMC simulation can be found in the literature (Aguilar and West, 2000, Chib et al., 2001, Lopes and Migon, 2002 and Tsai and Hotta, 2005).
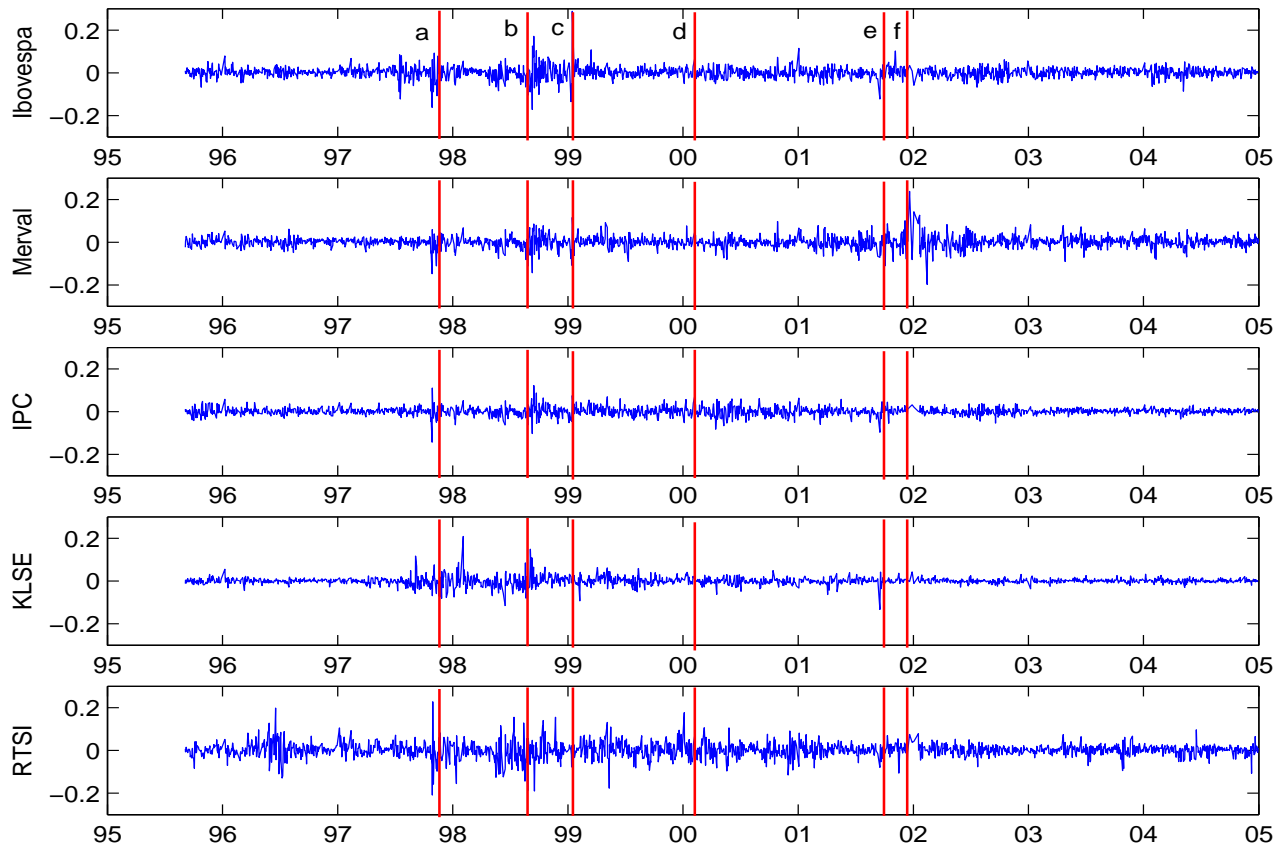

Figure 1: Daily returns of all indices. The vertical lines indicate the beginning of crisis periods: a the Asian crisis in second semester of 1997; $\mathbf{b}$ the Russian crisis in August of 1998, $\mathbf{c}$ the Brazilian crisis in 1999; $\mathbf{d}$ the Nasdaq fall in April of 2000; e terrorist attacks on September 11th, 2001; and $\mathbf{f}$ the Argentinian crisis at the end of 2001.

\section{The Data and Filtering}

The data set is composed of five stock exchange indices, namely, Ibovespa from Brazil, Merval from Argentina, IPC from Mexico, KLSE from Malaysia and RTSI from Russia, with daily data corresponding to the period from 09/05/1995 
to $12 / 30 / 2004$. We only considered the dates on which all the markets were open. This corresponded to 1,980 observations with a loss of approximately $14 \%$ of the original data. The data were obtained from the Yahoo! Finance website, http://finance.yahoo.com/, accessed on 02/05/2005.

We will adopt the compounded returns defined as $r_{t}=\ln \left(P_{t} / P_{t-1}\right)$, where $P_{t}$ is the stock market index value. Henceforth, these series will be simply referred to as returns.

The returns series are shown in figure 1. In the figure, the beginning of the main crises is indicated by vertical lines: a the Asian crisis in mid-October 1997, b the Russian crisis in August 1998, c the Brazilian crisis in 1999, d the Nasdaq fall in April 2000, e the terrorist attacks on September 11th, 2001, and $\mathbf{f}$ the Argentinian crisis at the end of 2001.

Table 2 presents some summary statistics for all series. The Russian index is the one with the greatest variance, followed by Latin American and Malaysian indices. The largest value of all the indices was achieved by Ibovespa and the smallest one by RTSI. The Ibovespa peak occurred in January 1999 and the minimum value in September 1998. The greatest value for the Merval series occurred in December 2001 and the smallest value in February 2002. For the KLSE, we had the maximum value in February 1998 and the minimum in September 2001, while for the IPC, the maximum value occurred in September 1998, the same period when the lowest value of Ibovespa occurred, and the minimum in October 1997. Finally, RTSI presented peaks and minimum in October 1997, coinciding with the lowest IPC value. Furthermore, these two extreme points of the RTSI index occurred on two consecutive days, October 28, 1997, respectively for the minimum and for the maximum values.

Table 2: Summary statistics of the returns before filtering

\begin{tabular}{lccccc}
\hline & Ibovespa & Merval & IPC & KLSE & RTSI \\
\hline mean $\left(10^{-4}\right)$ & 8.87 & 5.64 & 8.67 & -0.47 & 9.11 \\
maximum & 0.288 & 0.238 & 0.122 & 0.208 & 0.227 \\
minimum & -0.172 & -0.198 & -0.143 & -0.133 & -0.209 \\
variance $\left(10^{-4}\right)$ & 6.57 & 6.77 & 3.29 & 3.16 & 11.63 \\
kurtosis & 15.9 & 11.5 & 8.90 & 21.5 & 8.75 \\
asymmetry & 0.35 & 0.037 & -0.085 & 0.86 & -0.18 \\
\hline
\end{tabular}

The results in table 2 indicate that Merval and IPC returns distributions are more symmetric than the others, and that all distributions have heavier tails than the normal distribution.

During the Brazilian crisis, the volatility of Ibovespa, Merval and IPC indices were higher than the volatility of the other series. The volatility was lower before 
the Asian crisis and after the Argentinian crisis, except for Brazilian and Russian market returns. The volatility of Russian returns was already high at the beginning of the data set. We will refer to the period between the first (Asian) and last (Argentinian) crises as the between-crises period. The largest volatility was observed for Ibovespa, RTSI and Merval series. In general, the volatilities were larger in the between-crises period and smaller after the Argentinian crisis, except for the Merval index, which showed an increase in volatility. The RTSI volatility was already large before the Asian crisis, but increased thereafter. The KLSE volatility started to increase before the benchmark period showing that it started to be affected by the crisis in the other Asian countries.

\subsection{Filtering}

Although not shown, the serial correlation of all the returns are very small; except for Merval and IBOVESPA, all of them present statistically significant first-order correlation at 5\% level. Since the first-order correlation is statistically significant at $10 \%$ level even for these two series, we use an autoregressive filter of order one to all return series. The serial correlation of squared returns are small, but are statistically significant and persist for higher lags.

Comparing with the autocorrelation functions in the return series and in the squared return series before and after filtering, we noticed a large reduction in return autocorrelation, mainly in the first lag, as expected. The first autocorrelation is not signicant at $10 \%$ level for any series. Also, as expected, the mean can now be considered equal to zero (smaller than $10^{-9}$ ). The kurtosis decreased, but is still large, with maximum excess equal to 18.31 for the KLSE index and minimum excess equal to 6.10 for the RTSI index. The maximum and minimum values and the asymmetry coefficient remained almost the same. All the empirical analyses will be applied to these filtered data.

\section{Estimation}

In this section, we present the selection of the exponential smoothing parameters, the estimates of the multivariate GARCH and factorial models and the estimation of the correlation between the series. The analyses of the contagion estimates are presented in the next section.

\subsection{Exponential smoothing}

The correlation between each pair of series was estimated using the mgarch command and the ewma1 option ( further details in subsection 2.1) in S+FinMetrics. This means that the smoothing parameter is selected for each pair of series. 
The selected smoothing parameters are presented in table 3 . All the smoothing parameters are in the recommended interval of $(0.94,0.97)$, which is an indication that the method could be applied. The smallest value was 0.9446 for the Ibovespa-Merval pair, and 0.9679 for the Ibovespa-KLSE pair. Since the range of variation is not large, we would have very similar results if we had selected the same smoothing coefficient to estimate all the covariance matrices. The estimation of the correlation between the series is given in figure 2. The analysis of the correlations is done later, together with the other methodologies.

Table 3: Estimates of $\lambda$ for each pair of indices

\begin{tabular}{lcccc}
\hline & Merval & IPC & KLSE & RTSI \\
\hline Ibovespa & 0.9446 & 0.9549 & 0.9679 & 0.9507 \\
Merval & & 0.9618 & 0.9634 & 0.9557 \\
IPC & & 0.9667 & 0.9587 \\
KLSE & & & 0.9645 \\
\hline
\end{tabular}
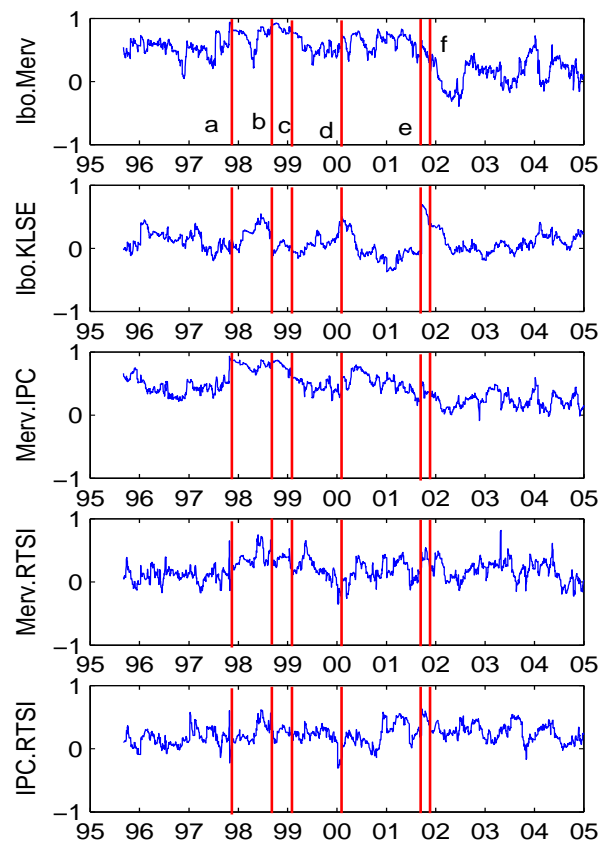
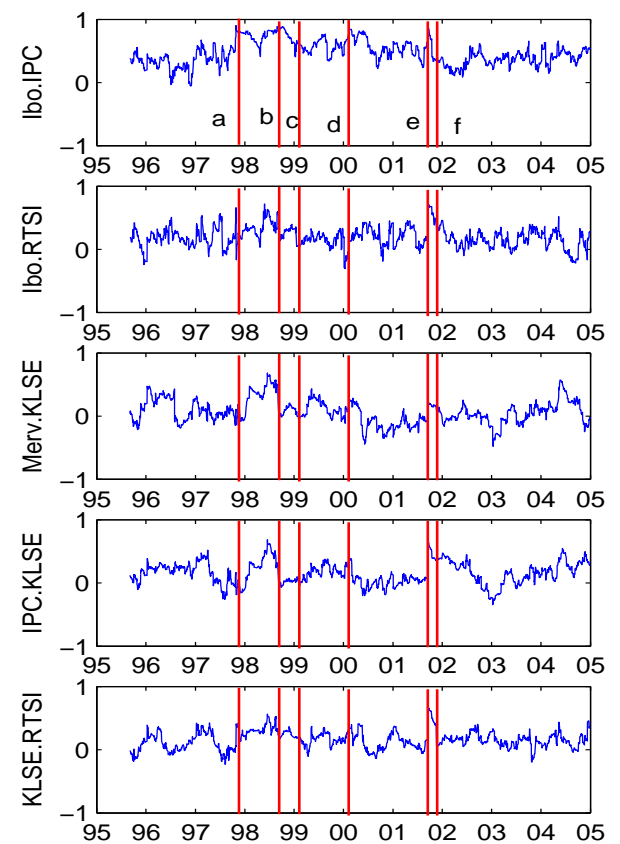

Figure 2: Correlations Estimates between each pair of indices given by exponential smoothing method. The vertical lines indicate the beginning of crisis periods: a the Asian crisis in second semester of 1997; $\mathbf{b}$ the Russian crisis in August of 1998, c the Brazilian crisis in 1999; d the Nasdaq fall in April of 2000; e terrorist attacks on September 11th, 2001; and $\mathbf{f}$ the Argentinian crisis at the end of 2001. 


\subsection{GARCH analysis}

\section{Estimation and specification testing}

All GARCH multivariate models were fitted using the Sheppard and Engle computational routines in Matlab. These routines are public and are available from the Sheppard website: http://www.kevinsheppard.com/research/uscd_garch.htm. We only present the results for BEKK and DCC models with orders $(1,1)$ and $(2,2)$ is for models with Gaussian disturbances. Student-t disturbances were also used, but the results are not reported because the results are worst than with Gaussian disturbances. Table 4 presents the values of the Bayesian Information Criteria (BIC) and the Akaike Information Criteria (AIC) for different models. We also estimated DCC models up to orders 4 , and BEKK models with $p=q=3$ and 4 , but the results were poorer compared to models with $(2,2)$ order. We only fitted BEKK models with the same order because we had problems of convergence with the Sheppard program when $p \neq q$.

Table 4: GARCH models with Gaussian disturbances

\begin{tabular}{lcccc}
\hline Model & $\ln \mathrm{L}(\theta) / \mathrm{T}$ & number of param. & AIC & BIC \\
\hline CCC $(1,1)$ & 12.787 & 25 & -50562 & -50422 \\
CCC $(2,2)$ & 12.817 & 35 & -50661 & -50465 \\
DCC $(1,1)$ & 12.816 & 17 & -50692 & -50597 \\
DCC (2,2) & 12.849 & 27 & -50801 & $\mathbf{- 5 0 6 5 0}$ \\
IDCC $(1,1)$ & 12.818 & 16 & -50703 & -50613 \\
IDCC $(2,2)$ & 12.826 & 26 & -50714 & -50569 \\
full BEKK $(1,1)$ & 12.800 & 65 & -50531 & -50167 \\
full BEKK $(2,2)$ & 12.904 & 115 & $\mathbf{- 5 0 8 4 3}$ & -50200 \\
diag.* BEKK $(1,1)$ & 12.744 & 25 & -50391 & -50251 \\
diag. BEKK $(2,2)$ & 12.832 & 35 & -50718 & -50522 \\
sca.* BEKK $(1,1)$ & 12.714 & 17 & -50287 & -50192 \\
sca. BEKK $(2,2)$ & 12.716 & 19 & -50292 & -50186 \\
\hline
\end{tabular}

According to the results presented in table 4, the BIC selected the DCC $(2,2)$ model while AIC selected the full BEKK $(2,2)$ model. The AIC selected DCC $(2,2)$ as the second best model. Out of the 27 parameters estimated for the DCC model, only four were not significant at $5 \%$ level, indicating that, besides providing a good adjustment, it is also parsimonious. On the other hand, in the BEKK model, 89 out of 115 parameters were not significant. Since the AIC criterion has the tendency to select models with an unnecessary number of parameters, it seems that the DCC $(2,2)$ model is the best one, but we will analyze both models. 
Table 5: Summary statistics of DCC $(2,2)$ (first line) and full BEKK $(2,2)$ (second line) models residuals

\begin{tabular}{lccccc}
\hline & Ibovespa & Merval & IPC & KLSE & RTSI \\
\hline kurtosis & 1.72 & 2.59 & 2.25 & 11.5 & 3.43 \\
& 1.96 & 2.49 & 1.61 & 7.18 & 2.60 \\
asymmetry & -0.050 & -0.0066 & -0.054 & -0.76 & -0.10 \\
& -0.041 & 0.019 & -0.035 & -0.34 & -0.021 \\
\hline
\end{tabular}

Table 5 presents some descriptive statistics of the standardized residuals of DCC $(2,2)$ while table 6 presents the results of the Ljung-Box test. The null hypothesis of absence of correlation in the squared residual series is rejected at $5 \%$ level only for the IPC index series. For all other cases the model appears to be effective in the elimination of serial correlation.

Table 6: Ljung-Box test for the DCC $(2,2)$ (first line) and full BEKK $(2,2)$ (second line) residuals and the squared model residuals

\begin{tabular}{lcccc}
\hline & \multicolumn{2}{c}{ residuals } & \multicolumn{2}{c}{ squared residuals } \\
& statistic & p-value & statistic & $p$-value \\
\hline Ibovespa & 18.66 & 0.54 & 11.36 & 0.94 \\
Merval & 19.17 & 0.51 & 42.30 & 0.003 \\
& 18.37 & 0.56 & 16.20 & 0.70 \\
IPC & 18.22 & 0.57 & 16.29 & 0.70 \\
& 10.81 & 0.95 & 38.29 & 0.09 \\
KLSE & 12.45 & 0.90 & 24.23 & 0.23 \\
\multirow{2}{*}{ RTSI } & 24.62 & 0.22 & 5.37 & 1.00 \\
& 21.85 & 0.35 & 14.84 & 0.79 \\
& 19.09 & 0.52 & 11.00 & 0.95 \\
& 19.04 & 0.52 & 9.46 & 0.98 \\
\hline
\end{tabular}

The specification tests were performed according to the presented methodology. With the first test, we checked whether there were any residual heteroskedasticity, and we used the other two tests to check for two types of asymmetry. The sets of variables used were:

$$
\begin{aligned}
\lambda_{1, t-1} & =\left\{\varepsilon_{1, t-\max (p, q)-1}^{2}, \ldots, \varepsilon_{5, t-\max (p, q)-1}^{2}\right\} \\
\lambda_{2, t-1} & =\left\{I_{\left(\varepsilon_{1, t-1}>0\right)}, \ldots, I_{\left(\varepsilon_{5, t-1}>0\right)}\right\} \\
\lambda_{3, t-1} & =\left\{\varepsilon_{1, t-1}^{2} I_{\left(\varepsilon_{1, t-1}>0\right)}, \ldots, \varepsilon_{5, t-1}^{2} I_{\left(\varepsilon_{5, t-1}>0\right)}\right\} .
\end{aligned}
$$

Table 7 presents the results of lack of specification tests for DCC $(2,2)$ and for the full BEKK $(2,2)$ models. None of the tests applied to all series were statistically significant at $5 \%$ level when applied to the residuals of the BEKK 
Juliana de P. Filleti et al.

Table 7: Tests of specification

\begin{tabular}{lcccccccc}
\hline & & \multicolumn{3}{c}{ DCC $(2,2)$} & & \multicolumn{3}{c}{ full BEKK $(2,2)$} \\
\cline { 3 - 5 } \cline { 8 - 9 } Indices & Moments & Statistics & p-value & d.f. & & Statistics & p-value & d.f. \\
\hline Ibovespa & $\lambda_{1}$ & 2.98 & 0.70 & 5 & & 2.97 & 0.70 & 5 \\
Merval & & 14.0 & $0.016^{*}$ & 5 & & 6.16 & 0.29 & 5 \\
IPC & & 6.36 & 0.27 & 5 & & 5.43 & 0.37 & 5 \\
KLSE & & 1.07 & 0.96 & 5 & & 6.10 & 0.30 & 5 \\
RTSI & & 1.73 & 0.88 & 5 & & 0.98 & 0.96 & 5 \\
\hline Ibovespa & $\lambda_{2}$ & 13.5 & $0.019^{*}$ & 5 & & 10.7 & 0.058 & 5 \\
Merval & & 10.8 & 0.056 & 5 & & 7.31 & 0.20 & 5 \\
IPC & & 9.01 & 0.11 & 5 & & 4.02 & 0.55 & 5 \\
KLSE & & 7.85 & 0.16 & 5 & & 7.39 & 0.19 & 5 \\
RTSI & & 9.02 & 0.11 & 5 & & 7.78 & 0.17 & 5 \\
\hline Ibovespa & $\lambda_{3}$ & 9.99 & 0.076 & 5 & & 5.67 & 0.34 & 5 \\
Merval & & 7.67 & 0.18 & 5 & & 2.26 & 0.81 & 5 \\
IPC & & 3.93 & 0.56 & 5 & & 4.62 & 0.46 & 5 \\
KLSE & & 8.23 & 0.14 & 5 & & 7.59 & 0.18 & 5 \\
RTSI & & 1.92 & 0.86 & 5 & & 1.46 & 0.92 & 5 \\
\hline
\end{tabular}

** statistically significant at $1 \%$ level; * significant at $5 \%$ level.

model, showing no lack of specification. The 15 tests applied to the DCC residuals were significant at $5 \%$ level only for the heteroskedasticity test $\left(\lambda_{1}\right)$ for the Merval index and effect of symmetry $\left(\lambda_{2}\right)$ for the Ibovespa index. Thus, we could consider that the model provided a good fit to the data.

Since the correlations estimated by both models are very similar and the estimates of the DCC model are smoother, we are going to present the DCC correlation results. The estimates are presented in figure 3 .

\subsection{Factorial analysis}

It is possible to use a maximum of two factor numbers because of the restriction given by $N(N+1) / 2>N k+N-k(k-1) / 2$. Ibovespa was the first selected factor because we have three Latin American countries and Brazil is the most important economy in the region. We considered a second factor, but the Markov simulation chain did not converge for all the entertained models. This is an indication that a second factor is not necessary. This result is expected in a certain way because we do not expect to find a close relationship between the Russian and Malaysian markets, except possibly during economic crises. The factorial model was estimated using a program written in $\mathrm{Ox}$. 

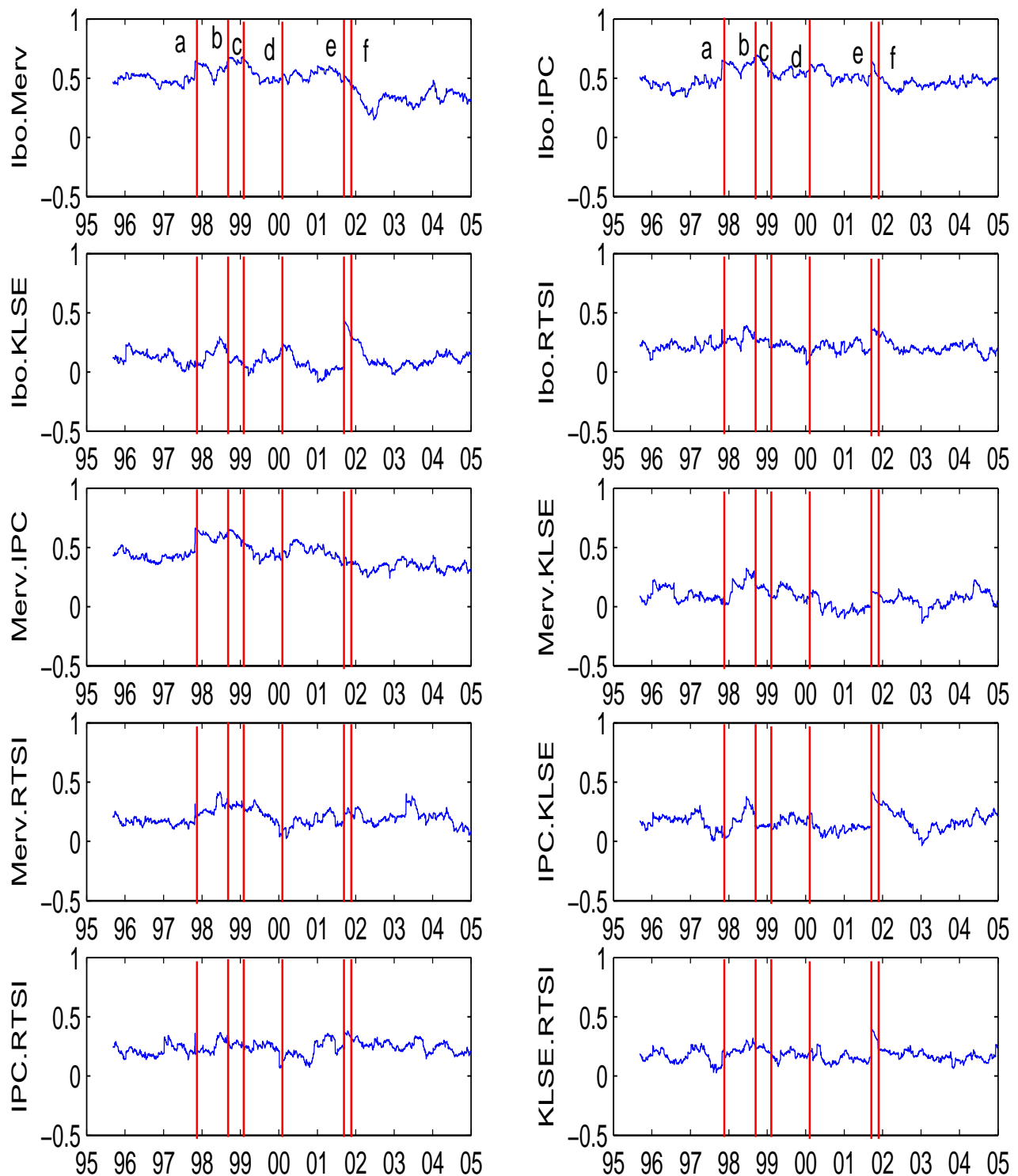

Figure 3: Correlations estimates to each pair of indices given by GARCH modelling DCC $(2,2)$ Gaussian. The vertical lines indicate the beginning of crisis periods: a the Asian crisis in second semester of 1997 ; $\mathbf{b}$ the Russian crisis in August of 1998, c the Brazilian crisis in 1999; d the Nasdaq fall in April of 2000; e terrorist attacks on September 11th, 2001; and $\mathbf{f}$ the Argentinian crisis at the end of 2001. 

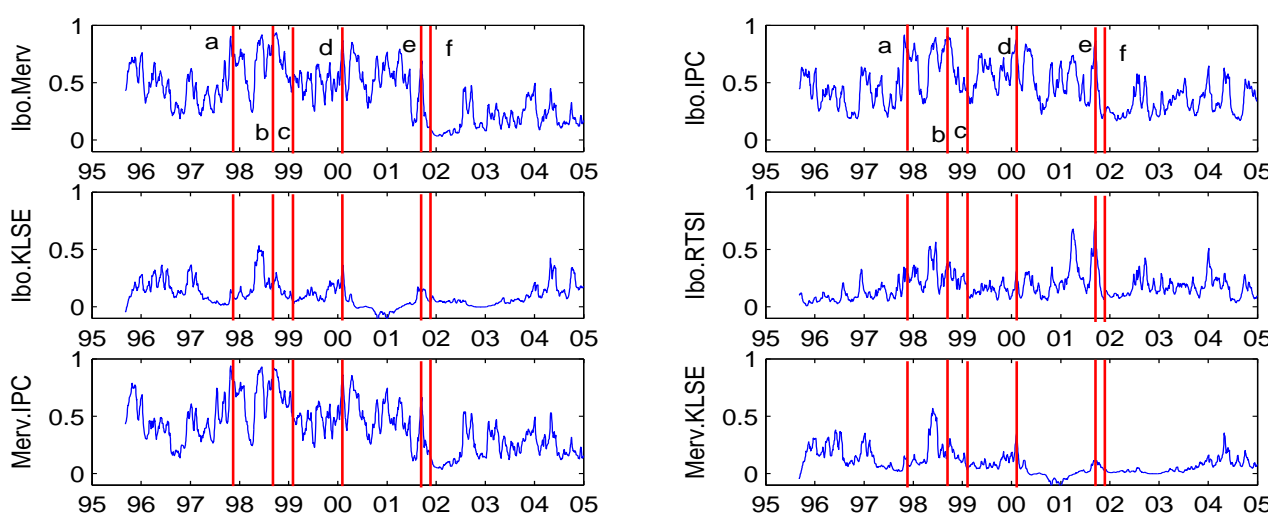

$\begin{array}{lllllllllll}95 & 96 & 97 & 98 & 99 & 00 & 01 & 02 & 03 & 04 & 05\end{array}$
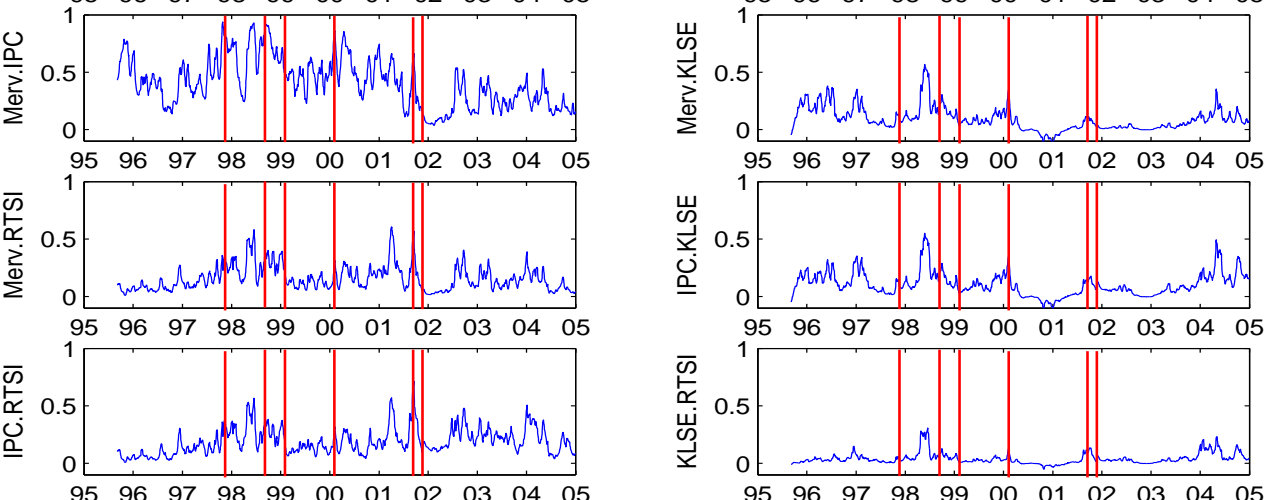

Figure 4: Correlation estimates to each pair of indices given by the factorial analysis with one factor. The vertical lines indicate the beginning of crisis periods: a the Asian crisis in second semester of 1997; b the Russian crisis in August of 1998, c the Brazilian crisis in 1999; d the Nasdaq fall in April of 2000; e terrorist attacks on September 11th, 2001; and $\mathbf{f}$ the Argentinian crisis at the end of 2001.

Table 8: Mean and standard deviation of the posterior distribution of the time invariant parameters of the factorial model

\begin{tabular}{lcccccc}
\hline & Factor & Ibovespa & Merval & IPC & KLSE & RTSI \\
\hline mean: $\mu^{f}$ & -8.783 & -8.563 & -8.581 & -9.033 & -9.221 & -7.493 \\
standard deviation: $\mu^{\varepsilon}$ & 0.219 & 0.134 & 0.191 & 0.198 & 0.286 & 0.153 \\
mean: $\phi^{f}$ & 0.958 & 0.943 & 0.946 & 0.956 & 0.988 & 0.942 \\
standard deviation: $\phi^{\varepsilon}$ & 0.013 & 0.022 & 0.018 & 0.011 & 0.005 & 0.011 \\
\hline
\end{tabular}


The estimation of the mean and standard deviation of the posterior distribution of the time-invariant parameters of the models are presented in table 8 . The smallest mean of the posterior distribution of the persistence $\phi$ was 0.946 (Merval) whereas the largest one was 0.988 (KLSE) (the persistence is defined by $\phi$ ). The estimation of the time-varying loading matrix and the common and specific log variances are not presented. The covariances and correlation matrices can be estimated by equation (2.12) and the estimation of the correlation presented in figure 4.
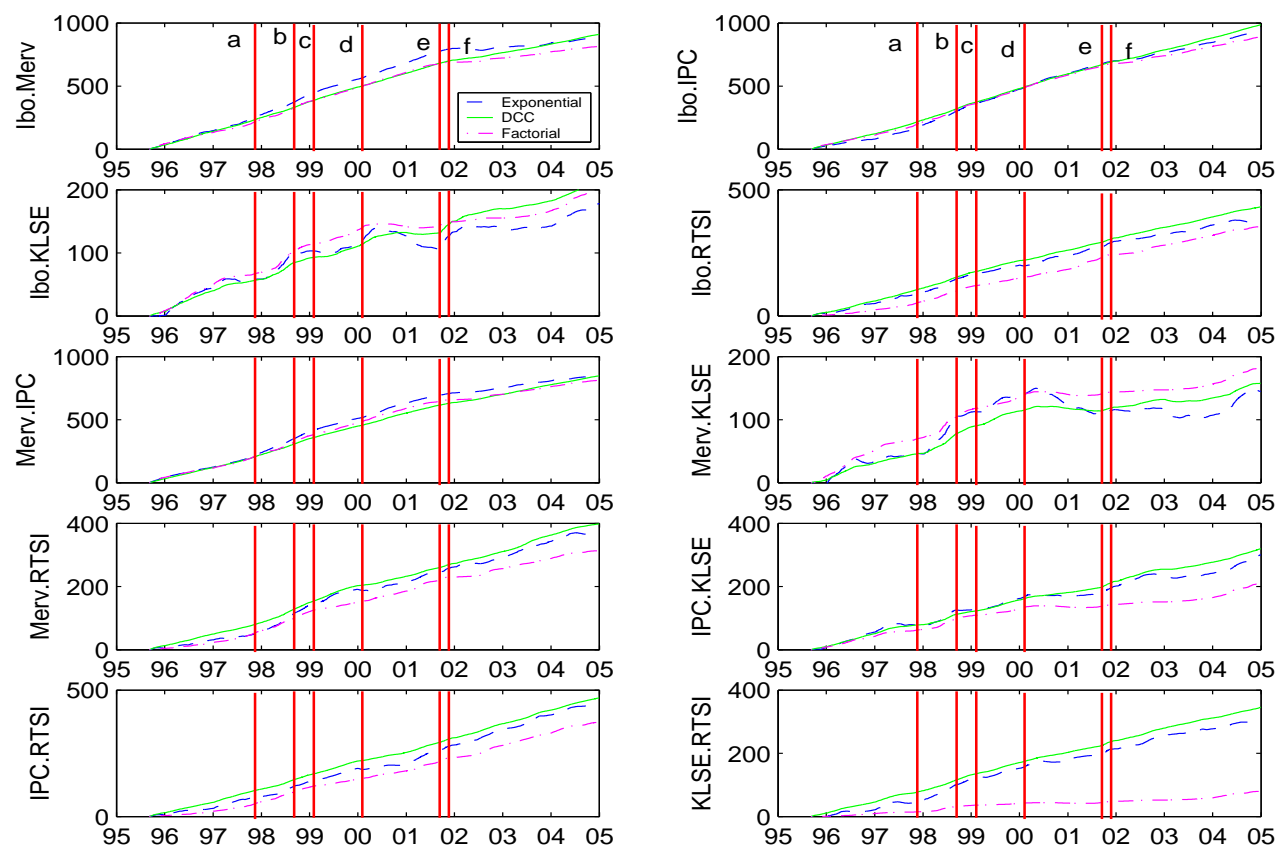

Figure 5: Correlation cumulative sum graph for each pair of indices. The vertical lines indicate the beginning of crisis periods: a the Asian crisis in second semester of 1997; b the Russian crisis in August of 1998, $\mathbf{c}$ the Brazilian crisis in 1999; d the Nasdaq fall in April of 2000; e terrorist attacks on September 11th, 2001; and $\mathbf{f}$ the Argentinian crisis at the end of 2001.

\section{Comparison of Results}

In this paper, we have shown three different methods to estimate the conditional correlation between the stock market return indices of five Emerging Markets: Brazil, Argentina, Mexico, Malaysia, and Russia. The estimated correlations were presented in the previous section. One way to assess the increase in correlation during crisis periods is through correlation cumulative sum graph. An 
increase in the slope in a period will indicate an increase in the correlation in this period. These graphs are shown in figure 5 . The results of the three estimation methods are very similar, except for the estimation of the correlation between KLSE and RTSI by the factorial method. In many cases, there was an increase in correlation after the Asian crisis. In two cases, there was a strong decrease in correlation after the Nasdaq fall (between the Malaysian index and the Brazilian and Argentinian indices). We also have a slight decrease in correlation between some markets after the Argentinian crisis.

Table 9 summarizes the results and presents the periods (a, Asian; b, Russian; c, Brazilian; d, Nasdaq; e, September 11th; and f, Argentina) and methods which identified the contagion (E, Exponential; G, Multivariate GARCH; and F, Factorial). We only considered indication of contagion when the correlation increased. Since there is no clear indication about the beginning of the Asian crisis, we consider that there is indication of contagion even when there was an increase in correlation before the Hong Kong market crash. In some cases, the increase in correlation does not occur immediately after the crisis, but there is a delay. In this case, we indicated it as a delayed contagion. We also indicated the cases where the increase in correlation is small. The results were often coherent between the three procedures, but sometimes they were not. Nevertheless, there is some agreement among all methods: in most cases, the contagion was detected only during the Asian crisis, indicating that this was the most significant crisis in our sample period. All the three methods detected contagion during the Asian crisis in the following pairs: Brazil and Argentina, Brazil and Mexico, Argentina and Mexico, and Argentina and Russia. During this period, the exponential method detected contagion between Argentina and Malaysia and between Mexico and Malaysia, both with delay, while the factorial method additionally detected a slight contagion between Brazil and Russia, and the GARCH method detected a delayed contagion between Argentina and Malaysia. Surprisingly, however, we did not detect any direct contagion between Malaysia and any of the other four countries through any of the methods. However, Forbes and Rigobon (2002) also did not find evidence of contagion from the Hong Kong stock market index and the same four countries. The exponential and GARCH methods detected contagion during the September 11th terrorist attack in the following pairs: Brazil and Malaysia, Brazil and Russia, Argentina and Malaysia, Mexico and Malaysia, and Malaysia and Russia. Additionally, during this period, the exponential method detected contagion between Argentina and Russia and the factorial method found contagion between Brazil and Russia. The Nasdaq fall was the only other crisis during which a slight contagion was detected, in this case, between Brazil and Malaysia, using the exponential method. 
Table 9: Summary of the contagion detected by the three methods presenting the crises (a, Asian; b, Russian; c, Brazilian; d, Nasdaq; e, September 11th; and $\mathrm{f}$, Argentina) and methods which identified the contagion (E, Exponential; G, Multivariate GARCH; and F, Factorial)

\begin{tabular}{|c|c|c|c|c|}
\hline Markets & Merval & IPC & KLSE & RTSI \\
\hline Ibovespa & $\mathrm{a}(\mathrm{E}, \mathrm{G}, \mathrm{F})$ & $\mathrm{a}(\mathrm{E}, \mathrm{G}, \mathrm{F})$ & $\begin{array}{c}\mathrm{d}\left(\mathrm{E}^{1}\right) \\
\mathrm{e}(\mathrm{E}, \mathrm{G})\end{array}$ & $\begin{array}{c}\mathrm{a}(\mathrm{F}) \\
\mathrm{e}\left(\mathrm{E}, \mathrm{G}^{1}, \mathrm{~F}^{1}\right)\end{array}$ \\
\hline Merval & $\cdots$ & $\mathrm{a}(\mathrm{E}, \mathrm{G}, \mathrm{F})$ & $\mathrm{a}\left(\mathrm{E}^{2}, \mathrm{G}^{2}\right)$ & $\begin{array}{c}\mathrm{a}\left(\mathrm{E}^{1}, \mathrm{G}^{1}, \mathrm{~F}^{1}\right) \\
\mathrm{e}(\mathrm{E})\end{array}$ \\
\hline IPC & $\ldots$ & $\cdots$ & $\begin{array}{c}a\left(E^{2}\right) \\
e(E, G)\end{array}$ & $\mathrm{a}\left(\mathrm{F}^{1}\right)$ \\
\hline KLSE & $\cdots$ & $\cdots$ & $\cdots$ & $\begin{array}{c}a(E) \\
e(E, G)\end{array}$ \\
\hline
\end{tabular}

${ }^{1}$ slight contagion, ${ }^{2}$ delayed contagion

None of the methods detected contagion between Brazil and Argentina during the Brazilian and Argentinian crises. This could be surprising because of the close relationship between these countries, but the markets were probably already expecting the Brazilian and Argentinian crises and could have taken hedging precaution. In general, we could say that the three methods produced similar results, although without complete agreement. There was more agreement between the exponential and GARCH methods.

Compared with similar analyses found in the literature, we have an agreement in most of the cases. Lopes and Migon (2002) used the factorial model to investigate the contagion between three Latin American countries, but included Chilean IPSA and the U.S. Dow Jones indices and did not consider the Russian and Malaysian markets. The analyzed period was a little different because they included the 1994 Mexican Tequila effect, but did not include the Argentinian crisis. Marçal and Valls Pereira (2005), on the other hand, used the multivariate GARCH models to study the contagion between three Latin American countries and Russia, but they worked with the sovereign debt bonds from January 1994 to December 2002. In these works, the authors also used the concept of shiftcontagion, but they considered that there was evidence of contagion whenever there was a shift in correlation, while we considered that there was evidence of contagion only when there was an increase in correlation. They also analyzed the contagion across countries even when the crisis had not originated in any of them. Lopes and Migon (2002) commented that there was evidence of contagion in Latin American countries during the Asian, Russian and Brazilian crises without pointing exactly when and where. However, by analyzing the results, one finds that correlation decreased during the Brazilian crisis whenever there was a shift in correlation. So, there is no contagion according to our definition, 
but there is indication of contagion in Brazilian, Argentinian and Mexican markets during the Asian crisis. Marcal and Valls Pereira (2005) reported evidence of contagion during the Asian and Russian crises, but not during the Brazilian and Argentinian crises. However, during the Russian crisis, there was in fact a decrease in correlation.

Thus, in general there is more agreement between our results and the results found in the literature, although the models were fitted to different series and different periods.

\section{Acknowledgments}

We thank Rodrigo Tsai for supplying the program to estimate the factorial model and Epifisma laboratory (UNICAMP). This work was partially supported by grants from CNPq, CAPES and FAPESP.

\section{References}

Aguillar, O. and West, M. (2000). Bayesian dynamic factor models and portfolio allocation. Journal of Business and Economic Statistics 18, 338-357.

Bae, K-H. Karolyi, G. A. and Stulz, R. M. (2000). A new approach to measuring financial contagion. NBER Mimeo 7913. Cambridge, Massachusetts.

Baig, T. and Goldfajn, I. (1998). Financial market contagion in the Asian crisis. IMF Working Paper No. 155.

Berndt, E., Hall, B., Hall, R. and Hausman, J. (1974). Estimation and inference in nonlinear structural models. Annals of Economic and Social Measurement 3, 653665 .

Bollerslev, T. (1990). Modelling the coherence in short-run nominal exchange rates: A multivariate generalized arch model. The Review of Economics and Statistics $\mathbf{7 2}$, $498-505$.

Boyer, B.H., Michael, S.G. and Loretan, M. (1999). Pitfalls in tests for changes in correlation. Working Paper No. WP 597R, Federal Reserve Board, International Finance Division, Washington.

Cappiello, L., Engle, R. F. and Sheppard, K. (2003). Asymmetric dynamics in the correlations of global equity and bond returns. European Central Bank - Working Papers Series, No. 204, January.

Chib, S., Nardari, F. and Sheppard. N. (2001). Markov chain Monte Carlo methods. Journal of Econometrics 108, 281-316. 
Corsetti, G., Pericoli, M. and Sbracia, M. (2002). Some contagion, some interdependence - more pitfalls in tests of financial contagion. C.E.P.R. Discussion Papers, n. 3310, April.

Dornbusch, R., Park, Y. C. and Claessens, S. (2001). Contagion: Why crises spread and how this can be stopped. In International Financial Contagion (Edited by Claessens, S., and Forbes, K.), 19-42. Springer.

Edwards, S. (1998). Interest rate volatility, capital controls, and contagion. NBER Working Paper No. 6756 (October), Cambridge, Massachusetts.

Engle, R. F. (2002). Dynamic conditional correlation: a simple class of multivariate generalized autoregressive conditional heteroskedasticity models. Journal of Business and Economic Statistics 20, 339-35

Engle, R. F. and Kroner, K. F. (1995). Multivariate simultaneous generalized arch. Econometric Theory 11, 122-150.

Engle, R. F. and Sheppard, K. (2001). Theoretical and empirical properties of dynamic conditional correlation multivariate garch. NBER Working Paper, Cambridge, n. 8554, October.

Fletcher, R. and Powell, M. J. D. (1963). A rapidly convergent descent method for minimization, Computer Journal 6, 163-168.

Forbes, K. J. and Rigobon, F. (2001). Measuring contagion: Conceptual and empirical issues. In International Financial Contagion (Edited by Claessens, S. and Forbes, K.), 43-66. Springer.

Forbes, K. J. and Rigobon, F. (2002). No contagion, only interdependence: measuring stock market comovements. The Journal of Finance 57, 2223-2261.

Geweke, J. and Zhou, G. (1996). Measuring the pricing error of the arbitrage pricing theory. Review of Financial Studies 9, 557-587.

Harvey, A. C., Ruiz, E. and Shephard, N. (1994). Multivariate stochastic variance models. Rev. Econ. Studies 61, 247-264.

King, M. and Wadhwani, S. (1990). Transmission of volatility between stock markets. Review of Financial Studies 51, 5-33.

Lopes, H. F. and Migon, H. S. (2002). Comovements and contagion in emergent markets: stock indexes volatilities. Case Studies in Bayesian Statistics VI, 285-300, Springer-Verlag.

Loretan, M. and English, W. B. (2000). Evaluation "correlation breakdowns" during periods of market volatility. Mimeo., Federal Reserve Board, Washington.

Marçal, E. F. and Valls Pereira, P. L. (2005). Testando a hipótese de contágio a partir de modelos multivariados de volatilidade. Anais do X Encontro Brasileiro de Finanças, São Paulo. (in Portuguese).

Newey, W. and McFadden D. (1994). Large sample estimation and hypothesis testing. In Handbook of Statistics, V4 (Edited by Engle E. and McFadden D.), 2113-2245. North-Holland. 
Pesaran, M. H. and Pick, A. (2005). Econometric issues in the analysis of contagion. CESifo Working Paper No. 1176.

Rigobon, R. (2001). Contagion: How to measure it? NBER Working Paper No. 8118, NBER, Cambridge, Massachusetts.

Tsai, R. and Hotta, L. K. (2005). Análise de séries temporais com covariâncias variando no tempo através de fatores com volatilidade estocástica. Revista de Matemática e Estatística 23, 1-20. (in Portuguese).

Wooldridge, J. M. (1990). A unified approach to robust, regression-based specification tests. Econometric Theory 6, 17-43.

Zivot, E. and Wang, J. (2005). Modeling Financial Time Series with S-Plus (2nd edition). Springer Verlag.

Received February 13, 2007; accepted June 15, 2007.

Juliana de Paula Filleti

P. O. Box 6016

FACAMP

13083-970 Campinas, SP, Brazil.

juledp@yahoo.com.br

Luiz K. Hotta

Institute of Mathematics, Statistics and Scientific Computing

University of Campinas

P. O. Box 6065

13083-970 Campinas, SP, Brazil.

hotta@ime.unicamp.br

Mauricio Zevallos

Institute of Mathematics, Statistics and Scientific Computing

University of Campinas

P. O. Box 6065

13083-970 Campinas, SP, Brazil.

amadeus@ime.unicamp.br 